\title{
Lyme Meningoradiculitis: Case Reports and Literature Review
}

\author{
Maier Smaranda1,2, Stirbu Nicoleta1,2, Bajko Zoltan¹,2, Moțățăianu Anca1,2, Țilea Brândușa1,2, \\ Alexandrescu Adrian ${ }^{1,2}$, Bălașa Rodica ${ }^{1,2}$ \\ 1 University of Medicine and Pharmacy Tirgu Mures, Romania \\ 2 Mures County Clinical Emergency Hospital, Romania
}

The clinical manifestations of Lyme disease are protean. The meningoradiculitis is a common and well-recognized complication of neuroborreliosis but can be easily misdiagnosed without a high degree of clinical suspicion, mainly if the tick bite is not present in the medical history. We report two cases of Lyme meningoradiculitis with excellent outcome after appropriate antibiotic therapy. In an endemic area in case of neurological manifestations suggestive for neuroborreliosis the serological testing for B. burgdorferi in serum and cerebrospinal fluid is imperative for the correct diagnosis.

Keywords: Lyme disease, Borrelia burgdorferi, meningoradiculitis

Received: 15 January 2016 / Accepted: 03 June 2016

\section{Introduction}

Lyme disease is the most common arthropod-borne infection in the Northern Hemisphere with an incidence of 9.7 cases per 100000 population in the United States (US). It is a multisystemic inflamatory disease caused by the three species of the spirochete Borrelia burgdorferi sensu lato (B. burgdorferi sensu stricto, B. afzelii and B. garinii), which is transmitted to human beings through bites of the infected Ixodes ticks $[1,2]$. The hematogenous dissemination will be followed by the systemic manifestations of the disease, caused by the involvment of the dermatologic, cardiac, neurologic and musculoskeletal systems. Lyme neuroborreliosis (LNB) will develop in about $8 \%$ of the patients with Lyme disease in US and $16-46 \%$ in Europe [1-3].

As in the case of another spirochete infection, Treponema pallidum, that causes syphilis, the neurological manifestations in LNB are protean, depending on the clincal stage at presentation [1]. The most frequent signs and symptoms of LNB in the early stages are lymphocytic meningitis, cranial nerve palsies and radiculopathy, while peripheral neuropathy, encephalomyelitis and mild encephalopathy are encountered in the late stages of the disease [4-7]. In Europe, the onset of LNB frequently is with painful lymphocytic meningoradiculitis, which is rarely found in US [2]. We present two cases of LNB manifested with meningoradiculitis.

\section{Case presentations}

\section{Patient 1}

A 20-year-old man, forest worker, with a negative medical

${ }^{*}$ Correspondence to: Nicoleta Stirbu

E-mail: degetar_nicoleta@yahoo.com history, presented in August, 2013, two weeks before admission radicular pain in the upper limbs, motor weakness in the right upper limb, followed, 2 days later, by weakness in the left upper limb and peripheral facial palsy on the left side after 1 week. He denied ever being bitten by a tick. The physical examination was unremarkable. The neurological examination at admission revealed: left peripheral facial palsy, brachial diparesis $1 / 5$ proximally and $3 / 5$ distally, diminished deep tendon reflexes in the upper limbs, atrophy of shoulder girdle muscle, scapulae alatae more significant on the left.

The usual laboratory examinations (complete blood count, blood glucose, lipid panel, urea, creatinine, sodium, potassium, aspartate aminotransferase, alanine aminotransferase and erythrocyte sedimentation rate (ESR) ) were normal. Negative results were obtained for antinuclear antibodies, anti-dsDNA antibodies, anticardiolipin antibodies, cryoglobulin levels, lupus cells, HIV-1 and HIV-2 antibodies and Veneral Disease Research Laboratory (VDRL).

Cerebrospinal fluid (CSF) analysis revealed elevated protein level $(613 \mathrm{mg} / \mathrm{l}$; reference interval (RI), $150-450 \mathrm{mg} /$ $\mathrm{dL}$ ), lymphocytic pleocytosis (406 leukocytes/3 microL, $92 \%$ lymphocytes; RI, $0-5 / 3 \mathrm{microL}$ ) and reduced glucose levels (44.9mg/dL; RI, 50-60mg/dL).

Serological testing for Borrelia in both serum and CSF was requested, but the patient refused another lumber puncture for CSF analysis. Serum IgM (125 UA/ml; RI $<18.0 \mathrm{UA} / \mathrm{ml})$ and $\mathrm{IgG}(240 \mathrm{UA} / \mathrm{ml} ; \mathrm{RI}<0.2 \mathrm{UA} / \mathrm{ml})$ Lyme titers were elevated.

The brain and cervical spine magnetic resonance imaging (MRI) without contrast administration were without pathological changes. Electromyographic examination was suggestive for a right cervical radiculopathy. It revealed normal motor conduction velocities in the left upper limb 
and normal sensory conduction velocities in both upper limbs, and neurogenic changes in right cervical myotomes.

Based on clincal and paraclinical examinations, a diagnosis of possible LNB was established and he was given treatment with oral doxycycline $200 \mathrm{mg}$ twice daily for 21 days. At follow-up visit, three month later, the symptoms had almost completely disappeared, the patient presented with a brachial diparesis $4 / 5$ proximally and $4+/ 5$ distally. After one year the neurological examination was normal.

\section{Patient 2}

A 78-year-old woman, with a history of hypertension and mitral and aortic valve stenosis, with poor adherence to antihypertensive drugs, was admitted in our clinic in November 2015 with a 2 -month history of bilateral occipital headache and intense, lancinating pain in upper limbs more severe overnight, associated with lethargy and progressive weakness in both upper limbs. She lives in a farm and she recalled an insect bite in August, 2015 followed by a periareolar erythema on her left breast. Neurological examination showed brachial diparesis $2 / 5$ proximally and $3 / 5$ distally, diminished deep tendon reflexes in upper limbs, indifferent bilateral plantar response, wasting of the shoulder girdle muscles and of the small muscles of the hands.

The brain MRI examination showed severe cerebral atrophy, with multiple lacunar infarcts. The MRI scan of her cervical spine, without contrast administration, was unremarkable, apart for some minor degenerative lesions of the vertebral bodies. The electromyographic examination revealed subacute neurogenic changes with bilateral active denervation of the cervical myotomes.

The complete blood count, ESR, blood chemistry and vitamin B12 level were in normal range. For VDRL and HIV we obtained negative results. Her CSF showed a raised protein level $(1252 \mathrm{mg} / \mathrm{dL}$; RI, $150-450 \mathrm{mg} / \mathrm{dL})$ and predominantly lymphocytic pleocytosis (780 leukocytes/3 microL with $85 \%$ lymphocytes; RI, 0-5leukocytes/microL). The titer of Borrelia IgM and IgG antibodies in serum ( IgM: 190UA/mL; RI <18UA/ml; IgG: 4.19UA/ $\mathrm{mL} ; \mathrm{RI}<0.2 \mathrm{UA} / \mathrm{mL}$ ) and CSF (IgM: $251 \mathrm{U}$; $\mathrm{RI}<9 \mathrm{U}$; IgG: $125 \mathrm{U} ; \mathrm{RI}<9 \mathrm{U})$ were elevated.

The diagnosis of LNB was estableshed and the patient was given treatment with intravenous cefotaxim (6g daily) for 15 days. At follow-up, 3 weeks later, her headache, fatigability and weakness in the upper limbs resolved completely.

\section{Discussion}

Lyme disease can be diagnosed in one of the three stages, depending on the duration of the infection. The first stage of the disease, early localized disease, occurs in the first month and typically manifests with a rash, erythema migrans, situated 7-14 $\mathrm{mm}$ from the tick bite site, accompanied by flu-like symptoms of fever, myalgia, arthralgia and headache. In the second stage, which occurs 1-4 month after an infected tick bite, the disease disseminates to other regions of the skin, nervous system, heart and joints and can present with a wide variety of symptoms. Most common neurological manifestations in this stage of the disease are meningitis, cranial nerve palsies, radiculopathy, and less common are found acute myelitis, encephalitis, cerebellar ataxia or mononeuritis multiplex. The third stage, late Lyme disease, occurs after months or years from the tick bite and typically manifests through neurological symptoms (peripheral neuropathy, chronic encephalopathy and encephalomyelitis) and large joints arthritis [4,8-10].

Given the great variety of clinical manifestations that can occur, the diagnosis of Lyme disease is sometimes difficult because the patient does not recall the tick bite (Ixodes ticks are very small and may fall off after feeding) and erythema migrans occurs in only $50-70 \%$ of patients. Both our patients were diagnosed as having possible (case 1) and definite (case 2) stage II Lyme disease due to the patient history, neurological manifestations, serological results and the presence of the erythema migrans in the second patient $[2,4,10]$.

In a study conducted in Denmark on a number of 13923 people, LNB has been confirmed in 533 of the patients. This study demonstrated that LNB has a seasonal variation, the lowest number of cases was found between February and May, and the highest number of cases in August and September [11]. Our patients presented neurological signs of LNB in August and September respectively.

The clinical picture of LNB is different between US and Europe. Even if meningoradiculitis is the most common manifestation of LNB in Europe, its incidence in the US is approximately $3 \%[12,13]$. The explanation for the different clinical manifestations of LNB between the two continents can be given by the different genospecies, B. garinii and B. bavariensis (tipicaly identified in patients with meningoradiculitis) are endemic in Europe but not in US [12]. The triad of meningitis, cranial neuritis (facial nerve being the most commonly affected cranial nerve) and radiculoneuritis, which are the neurological symptoms encountered in our first patient, is known in Europe as Garin-Bujadoux-Bannwarth syndrome [14]. This syndrome was first described in 1922 in Lyon in a 58-year-old man with a history of tick bite, followed three weeks later by erythema at the site of the bite and intense sciatic pain. He developed neuropathic pain in both lower limbs and right upper limb and weakness in the right shoulder. CSF analysis revealed pleocytosis and elevated protein level. $\mathrm{He}$ was treated with neoarsphenamine and recovered $[15,16]$.

According to the European Federation of Neurological Sciences guidelines, it is required to meet two of the following three criteria in order to diagnose possible LNB, and three for the definitive diagnosis: 1. Neurological symptoms; 2. CSF lymphocytic pleocytosis; 3. Detection of intrathecally produced B. burgdorferi-specific antibodies, or identification of Borrelia in the CSF by polymerase chain reaction (PCR) or culture if the symtoms started in the last 6 weeks $[8,17]$. Our second patient met the criteria for a definite diagnosis of LNB, while the first case met only two of this three criteria, meaning a possible diagnose of LNB. 
When Lyme disease is suspected, the serological diagnosis is usually a two-step method to detect antibodies to B burgdorferi. A sensitive enzyme-linked immunosorbent assay (ELISA) or indirect florescent antibody assay are used initially, and a positive or equivocal initial test is then commonly confirmed by immunoblot or Western blot. If the ELISA is negative, the sample does not need any further testing $[2,18]$. When LNB is suspected, CSF analysis for intrathecal production of antibodies to B Burgdorferi is useful for the diagnosis, but not enough because it can highlight a previous infection. It requires the presence of inflammatory cells in CSF (usually lymphocytes), often accompanied by high levels of proteins $[2,19]$. Intrathecal anti-Borrelia antibody index has a $95 \%$ specificity and 75\% sensitivity for the diagnosis of LNB.(1) Another test used for the diagnosis of LNB is PCR, but it has a lower sensitivity. B. Burgdorferi can also be identified by direct microscopy in biopsies, electron microscopy and by culture, but these tests have a lower accessibility $[2,18]$.

Even if the diagnosis of LNB is sometimes difficult to perform and requires a high degree of suspiciousness, the treatment protocols are very well defined and easy to follow [12]. European guidelines published in 2010, recommended as first-line treatment in case of LNB with peripheral manifestations, including meningitis, oral doxycycline (200 mg daily) and intravenous ceftriaxone ( $2 \mathrm{~g}$ daily) for 14 days (level A). For patients with CNS manifestations, treatment with intravenous ceftriaxone ( $2 \mathrm{~g}$ daily) for 14 days is the optimal therapeutic option and for late LNB (symptom duration $>6$ months) a 3 weeks treatment regimen is recommended (good practice points). Promising responses were obtained with 10-28-days regiments of intravenous penicillin ( 20 million units daily) and intravenous cefotaxime $(3 \times 2 \mathrm{~g}$ daily) $[12,20]$. A norwegian study conducted on 102 patients with LNB, demonstrated that a 14 days treatment with oral doxycycline (200mg daily) is not inferior to intravenous ceftriaxone $(2 \mathrm{~g}$ per day for 14 days) [21].

Recent studies have shown that if proper treatment is performed, at one year follow-up, only $16-28 \%$ of the patients still have symptoms, most often discrete. Delaying the treatment administration can lead to persistent symptoms, reflecting irreversible damage $[12,22,23]$.

\section{Conclusion}

The meningoradiculitis is a common complication of Lyme disease, the most frequent, completely curable, zoonoses in our region. Due to the great variability in clinical manifestations the early diagnosis requires a high degree of suspiciousness. An appropriate antibiotic treatment can lead to a full recovery in most patients.

\section{Acknowledgments}

This study was supported by the internal research Grant of The University of Medicine and Pharmacy Targu Mures, Grant Number 16171/17.11.2014.

\section{Conflict of interest}

Nothing to disclose

\section{References}

1. Ruxandra Calin, Adriana Hristea, Radu Tanasescu (2012). Central Nervous System Involvement in Lyme Disease - Making the Diagnosis and Choosing the Correct Treatment, Miscellanea on Encephalopathies, Radu Tanasescu (Ed.), ISBN: 978-953-51-0499-5, InTech, DOI: $10.5772 / 37669$.

2. Hildenbrand P, Craven DE, Jones R, Nemeskal P. Lyme neuroborreliosis: manifestations of a rapidly emerging zoonosis. AJNR Am J Neuroradiol. 2009;30(6):1079-87. doi: 10.3174/ajnr.A1579.

3. Blanc F, Gebly. Neurologic and psychiatric manifestations of Lyme disease. Medecine et Maladies Infectieuses 2007;37(7-8):435-445.

4. Zhang Y, Lafontant G, Bonner FJ Jr. Lyme neuroborreliosis mimics stroke: a case report. Arch Phys Med Rehabil. 2000;81(4):519-521.

5. Garcia-Monco JC, Benach Jl. Lyme neuroborreliosis. Ann Neurol. 1995;37:691-702.

6. Reik L, Steere AC, Bartenhagen NH, Shope RE, Malawista SE. Neurologic abnormalities of Lyme disease. Medicine (Baltimore). 1979;58(4):281-294.

7. Steere AC. Lyme disease. N Engl J Med 2001;345(2):115-125.

8. Subedi S, Dickeson DJ, Branley JM. First report of Lyme neuroborreliosis in a returned Australian traveller. Med J Aust. 2015;203(1):39-40.

9. Stanek G, Strle F. Lyme borreliosis. Lancet. 2003;362(9396):16391647.

10. Rahn DW, Felz MW. Lyme disease update. Current approach to early, disseminated, and late disease. Postgrad Med. 1998;103(5):51-4,57-9,63-4passim.

11. Dessau RB, Espenhain L, Mølbak K, Krause TG, Voldstedlund M. Improving national surveillance of Lyme neuroborreliosis in Denmark through electronic reporting of specific antibody index testing from 2010 to 2012. Euro Surveill. 2015;20(28).pii:21184.

12. Tobias A Rupprecht, Volker Fingerle. Neuroborreliosis: pathogenesis, symptoms, diagnosis and treatment. Future Neurology. 2011;6(2):273289. DOI: $10.2217 /$ fnl.10.89.

13. Jassam YN, Thaler DE. Lyme meningo-radiculitis responsive to oral doxycycline therapy in the USA. Oxf Med Case Reports. 2014;2014(9):162-163. doi: 10.1093/omcr/omu061. eCollection 2014.

14. Halperin JJ. Nervous system Iyme disease: is there a controversy? Semin Neurol. 2011;31(3):317-324. doi: http://dx.doi. org/10.1055/s-0031-1287652.

15. Halperin JJ. Chronic Lyme disease: misconceptions and challenges for patient management. Infect Drug Resist. 2015;8:119-28. doi: 10.2147/ IDR.S66739. eCollection 2015.

16. Garin C, Bujadoux A. Paralysie par les tiques. (tick paralysis). J Med Lyon. 1922;71:765-767.

17. Mygland $\AA$, Ljøstad $U$, Fingerle $V$, et al. EFNS guidelines on the diagnosis and management of European Lyme neuroborreliosis. Eur J Neurol. 2010;17:8-16,e1-4.

18. Aguero-Rosenfeld ME, Wang G, Schwartz I, Wormser GP. Diagnosis of lyme borreliosis. Clin Microbiol Rev. 2005;18(3):484-509.

19. Hengge UR, Tannapfel A, Tyring SK, Erbel R, Arendt G, Ruzicka T. Lyme borreliosis. Lancet Infect Dis. 2003;3(8):489-500.

20. MyglandA, LjøstadU, FingerleV, etal. EuropeanFederation of Neurological Societies. EFNS guidelines on the diagnosis and management of European Lyme neuroborreliosis. Eur J Neurol. 2010;17(1):8-16,e1-4. doi: 10.1111/j.1468-1331.2009.02862.x.

21. Ljøstad U, Skogvoll E, Eikeland R, et al. Oral doxycycline versus intravenous ceftriaxone for European Lyme neuroborreliosis: a multicentre, non-inferiority, double-blind, randomised trial. Lancet Neurol. 2008;7(8):690-695. doi: 10.1016/S1474-4422(08)70119-4.

22. Eikeland R, Ljøstad U, Mygland A, Herlofson K, Løhaugen GC. European neuroborreliosis: neuropsychological findings 30 months post-treatment. Eur J Neurol. 2012;19(3):480-487. doi: 10.1111/j.14681331.2011.03563.x.

23. Ljøstad U, Mygland A. Remaining complaints 1 year after treatment for acute Lyme neuroborreliosis; frequency, pattern and risk factors. Eur J Neurol. 2010;17(1):118-123. doi: 10.1111/j.1468-1331.2009.02756.x. 